\title{
Le tourisme international en Tunisie : vers de nouvelles formes et la réorganisation de l'espace touristique
}

Mohamed Souissi

\section{CpenEdition}

Journals

Édition électronique

URL : http://journals.openedition.org/cdg/2310

DOI : $10.4000 /$ cdg. 2310

ISSN : 2107-7266

Éditeur

UMR 245 - CESSMA

Référence électronique

Mohamed Souissi, «Le tourisme international en Tunisie : vers de nouvelles formes et la réorganisation de l'espace touristique », Carnets de géographes [En ligne], 1 | 2010, mis en ligne le 01 octobre 2010, consulté le 24 septembre 2020. URL : http://journals.openedition.org/cdg/2310 ; DOI https://doi.org/10.4000/cdg.2310

Ce document a été généré automatiquement le 24 septembre 2020.

\section{(c)}

La revue Carnets de géographes est mise à disposition selon les termes de la Licence Creative Commons Attribution - Pas d'Utilisation Commerciale - Pas de Modification 4.0 International. 


\title{
Le tourisme international en Tunisie : vers de nouvelles formes et la réorganisation de l'espace touristique
}

\author{
Mohamed Souissi
}

\section{Problématique et méthodes de recherche}

1 Les choix thématiques de cette thèse correspondent à une volonté de comprendre l'organisation et les limites du tourisme tunisien et d'étudier les possibilités de réorganiser l'espace touristique à travers le développement de nouvelles pratiques comme : le tourisme culturel, saharien, de santé (thalassothérapie et thermalisme), axé sur la nature (tourisme vert), ou encore le tourisme de sports et de loisirs (golf et plaisance).

2 Le travail de collecte de données auprès des acteurs publics et privés a été complété par une enquête par questionnaire auprès de 1032 touristes étrangers séjournant en Tunisie. C'est dans ce contexte que s'inscrit cette thèse qui consiste, à l'échelle de la Tunisie et à travers les principales composantes du système touristique, à poser les questions suivantes :

- Comment s'organise cette activité dans l'espace?

- Quels sont les contraintes et les enjeux liés au tourisme balnéaire?

- Comment se positionnent les nouvelles formes de tourisme?

- Quelles sont les perspectives d'une nouvelle organisation de l'espace touristique tunisien?

\section{Principaux résultats de la thèse}

Le tourisme en Tunisie est un facteur de développement économique, social et élément essentiel de l'organisation de l'espace. Ses performances sont le résultat des stratégies 
de l'État tunisien et des investisseurs privés, acteurs principaux dans l'organisation du système touristique. L'organisation spatiale du tourisme en Tunisie, se manifeste, essentiellement, par la présence des espaces balnéaires au sein de grandes villes littorales avec une très forte concentration des équipements, des pratiques touristiques et des taux d'urbanisation.

4 Le tourisme en Tunisie constitue un système contrôlé par les voyagistes étrangers dans un espace méditerranéen à forte concurrence touristique. Les tours opérateurs et les agences de voyages des pays européens occupent une position stratégique dans la gestion de l'activité touristique et constituent les deux acteurs principaux de la chaîne de fabrication et de distribution des voyages vers la Tunisie. Le groupe Nouvelles Frontières est l'exemple d'une intégration verticale d'un tour opérateur français dans le système touristique tunisien. L'étude de ce voyagiste a bien montré qu'il contrôle parfaitement les composantes essentielles du produit touristique en amont.

5 Lors de l'analyse du positionnement du tourisme tunisien dans son environnement méditerranéen, nous avons constaté que la Tunisie se caractérise par une forte dépendance vis-à-vis de ses principaux marchés émetteurs, et une offre touristique moins diversifiée que celles de ses concurrents directs (Maroc, Égypte, Grèce et Turquie).

6 En Tunisie, des nouvelles formes de tourisme sont faiblement intégrées dans le système touristique existant. L'étude quantitative de la fréquentation des sites culturels en Tunisie et les analyses des brochures publicitaires des voyagistes étrangers ont permis de montrer que seuls quelques sites sont intégrés dans cette forme de pratique touristique, essentiellement l'Amphithéâtre d'Eljem, le site de Carthage, la Médina de Tunis et le musée de Bardo (Tunis). L'étude du tourisme saharien montre que ce produit dépend du pôle balnéaire Djerba-Zarzis qui contrôle l'espace touristique dans les régions sahariennes par le biais des circuits organisés. Il ressort de l'étude de la thalassothérapie que ce produit joue un rôle important dans le renforcement de l'attractivité du tourisme littoral en basse saison touristique.

7 Les enquêtes de terrain auprès des acteurs publics et privés en Tunisie, et auprès des voyagistes européens (Paris, Bruxelles et Genève) ont permis de montrer que la pratique du tourisme vert se manifeste par l'organisation de circuits ornithologiques et de randonnées équestres et pédestres dans les régions montagneuses de Kroumirie, autour de Tabarka-Ain Draham et dans le Haut Tell, autour de la région d'El Kef. Par contre, notre étude des parcs nationaux a révélé qu'à l'exception de quelques formes actives de tourisme comme les randonnées équestres la marche et le camping pratiquées dans certains parcs, notamment Ichkeul, il n'y a pas aujourd'hui de parc qui se distingue remarquablement par cette forme de pratiques touristiques.

8 Concernant le tourisme de sport et de loisir (le golf et la plaisance), nous avons constaté que la clientèle de cette forme de tourisme s'intéresse également aux circuits culturels et aux randonnées dans le Sahara, ce qui constitue un atout pour le tourisme tunisien.

Quelles sont les possibilités d'une nouvelle organisation spatiale du tourisme tunisien? Nous avons élaboré un travail cartographique qui propose une nouvelle approche géographique de l'organisation spatiale du tourisme en Tunisie. Les cartes proposent le développement de nouveaux espaces susceptibles de diversifier le produit touristique tunisien, comme ceux axés sur : le tourisme culturel, le tourisme de nature orienté vers 
les randonnées et vers les parcs nationaux, et le tourisme saharien orienté vers la visite des oasis et les randonnées dans le désert.

\section{Conclusion}

Nous avons tenté à travers ce travail d'analyser les composantes et les contraintes du système touristique tunisien et d'étudier les nouvelles formes de tourisme ainsi que leur impact sur l'organisation de l'espace. Notre modeste contribution, qui a pris pour cadre géographique le territoire national, pourrait initier d'autres approches relatives aux nouvelles formes de tourisme à l'échelle régionale ou locale, qui peuvent en découler. Et c'est vers ces problématiques que nous envisageons d'orienter nos prochaines recherches.

\section{Fiche informative}

\section{Discipline}

Géographie

\section{Directeur}

Guy Chemla

\section{Université}

Université Paris-Sorbonne (laboratoire Espace, Nature et Culture)

Membres du jury de thèse, soutenue en mars 2007

- M. Adnane Hayder, Professeur à l'université de Tunis

- M. Abdallah Cherif, Professeur à l'université de la Manouba (Tunis)

- M. Jean-René Bertrand, Professeur à l'université du Maine (Le Mans)

- M. Michel Carmona, Professeur à l'université de Paris-Sorbonne (Paris 4)

- M. Guy Chemla, Professeur à l'université de Paris-Sorbonne (Paris 4)

\section{Situation professionnelle actuelle}

Enseignant-chercheur à l'Université de Sousse (Tunisie), faculté des Lettres et des Sciences Humaines, département de géographie

Contact de l'auteur

mohamedsouissi2114[at]yahoo.fr

\section{INDEX}

Thèmes : Carnets de soutenances 